ARTIGO ORIGINAL ORIGINAL ARTICLE

\section{Farmacoeconomia: gastos com análogos de insulina adquiridos por meio de judicialização em um município do estado do Pará, Brasil, no ano de 2016}

\author{
Pharmacoeconomics: expenses with analogues of insulin acquired \\ by judicialization in a county from the state of Pará, Brazil, in 2016 \\ Priscila de Nazaré Quaresma Pinheiro', João Victor Moura Garcia², Érica de Tassia \\ Carvalho Cardoso ${ }^{3}$, Diandra Araújo da Luz ${ }^{4}$, Valdenira Gonçalves da Silva ${ }^{5}$ \\ DOI: $10.21115 /$ JBES.v11.n1.p42-8
}

\section{Palavras-chave:}

farmacoeconomia, judicialização da saúde, insulina, insulina de ação prolongada, medicamentos essenciais

\section{Keywords:}

pharmacoeconomics, health judicialization, insulin, prolonged action insulin, essential medicines

\section{RESUMO}

Objetivo: Analisar os gastos com insulinas análogas de ação rápida e longa, no município de Belém/PA, no ano de 2016. Métodos: Trata-se de um estudo descritivo, quantitativo de farmacoeconomia, relacionado aos custos de insulinas análogas. A pesquisa foi realizada na Secretária Municipal de Saúde de um município do estado do Pará, por meio da análise das notas de empenho referentes à aquisição de insulinas análogas no ano de 2016. Os dados foram tabulados no Microsoft ${ }^{\circ}$ Excel 2010, no qual também foram geradas tabelas e gráficos para melhor interpretação das informações coletadas. A presente pesquisa não envolveu a participação de seres humanos e nem a utilização de dados secundários, por isso não houve a necessidade de submissão ao Comitê de Ética em Pesquisa (CEP). Resultados: Durante o ano de 2016, foram disponibilizadas 15 variedades de apresentação de análogos de insulinas. Neste ano, foram realizadas 10 compras de insulinas comprovadas por meio de empenho, resultando num total de 30.450 frascos de insulina, que gerou uma despesa extra de $R \$ 1.857 .778,00$ ao município. Em relação à quantidade comprada e ao custo de cada insulina análoga, a insulina Glargina liderou o ranking em ambas as variáveis, obtendo 12.650 frascos comprados e custo total de R\$967.970,00. Conclusão: Mesmo com a recente inclusão nas listas-padrão de algumas insulinas análogas que devem ser disponibilizadas pelo Sistema único de Saúde (SUS), essa ação ainda não ocorre de maneira efetiva no território brasileiro, evidenciado pelos gastos significativos com a compra de insulinas análogas por meio de judicialização.

\begin{abstract}
Objective: To analyze the costs of fast and long-acting analogues of insulin in Belém/PA, from 2016. Methods: This is a descriptive and quantitative study of pharmacoeconomics, related to the analogues of insulins cost. The research was realized at the Municipal Health Secretary from a county in the state of Pará, through analysis of the commitment notes regarding the acquisition of analogous of insulins from the year of 2016. The data were charted in Microsoft ${ }^{\circledR}$ Excel 2010, which also generated another's tables and graphs for the information collected better interpretation. The present research did not involve the human beings participation or even use of secondary data, so there was no need to submit to the Research Ethics Committee (REC/CEP). Results: During the year 2016, 15 presentation varieties of analogous of insulin were made available. This same year, 10 purchases of proven insulin were performed through a commitment, resulting in a total of 30.450 bottles of
\end{abstract}

Recebido em: 04/08/2018 Aprovado para publicação em: 24/02/2019

1. Mestrado em Patologia das Doenças Tropicais; docente da Universidade da Amazônia (Unama); farmacêutica da Secretária Municipal de Saúde (Sesma), Belém, PA, Brasil.

2. Acadêmico de Bacharelado em Enfermagem da Universidade da Amazônia (Unama), Belém, PA, Brasil.

3. Mestrado em Neurociência e Biologia celular; docente da Universidade da Amazônia (UNAMA), Belém, PA, Brasil.

4. Mestrado em Ciências Farmacêuticas; docente da Universidade da Amazônia - UNAMA. Belém, PA, Brasil.

5. Especialista em Gestão da Assistência Farmacêutica; farmacêutica da Secretária Municipal de Saúde (Sesma), Belém, PA, Brasil.

Nome da instituição onde o trabalho foi realizado: Secretária Municipal de Saúde (Sesma), Belém, PA, Brasil.

Financiamento: $O$ presente estudo obteve autofinanciamento dos pesquisadores envolvidos em todo o período de sua realização. Conflito de interesse: Este artigo é original, resultado da pesquisa realizada durante a formação acadêmica de Bacharelado em Enfermagem e não teve nenhum financiamento de instituição pública, privada ou órgão de fomento. Sendo assim, não há potenciais conflitos de interesse.

Autor correspondente: João Victor Moura Garcia. Av. Alcindo Cacela, 287, Umarizal, Belém, PA, Brasil. CEP: 66060-902.

Telefone: (91) 98129-3551. E-mail: joaovgarcia98@gmail.com 
insulin, which generated an extra expense of R\$1,857.778.00 to the county. Regarding the quantity purchased and the cost of each analogous insulin, Glargina insulin led the ranking in both variables, obtaining 12.650 bottles purchased and $\mathrm{R} \$ 967,970.00$ of total cost. Conclusion: Despite the recent inclusion at the standard lists of some analogues of insulins that should be made available by the Single Health System (SHS/SUS), this action still does not occur effectively in Brazil, evidenced by the significant expenses with the purchase of analogues of insulins through a judicial process (public civil action).

\section{Introdução}

Em condições normais, a glicose é encontrada em quantidade suficiente no sangue, com o intuito de servir como "combustível" para a geração de energia e a realização de funções orgânicas. A maioria dessa glicose advém da absorção de alimentos ingeridos no trato gastrointestinal e da formação de glicose pelo fígado. A diabetes mellitus (DM) é uma doença metabólica crônica, caracterizada pela deficiência total ou parcial da produção/secreção e/ou na ação da insulina pelo pâncreas, resultando em um estado de hiperglicemia, sendo classificada em DM tipo 1, DM tipo 2 e DM gestacional (Brasil, 2014; Smeltzer \& Bare, 2014).

Segundo a International Diabetes Federation (IDF), em 2015 aproximadamente 415 milhões de pessoas em todo o mundo conviviam com a DM, representando $8,8 \%$ da população mundial; desse total, aproximadamente 14,3 milhões de pessoas residiam no Brasil, sendo este considerado o quarto maior país com incidência da doença. Para o ano de 2040, é esperado a nível global que a DM atinja cerca de 642 milhões de pessoas, demonstrando aumento de 35\% (IDF, 2015; Henriques, 2016).

Em 1998, um estudo realizado em diversos países do mundo, inclusive no Brasil, estimou que entre 1995 e 2025 houvesse um aumento de $35 \%$ no número de casos existentes de diabetes nas pessoas com 20 ou mais anos de idade em todo o mundo. Em 1995, o Brasil, tinha uma população com cerca de 4,9 milhões de adultos diabéticos, enquanto para 2025 a estimativa aponta cerca de 11,6 milhões de diabéticos; em nível global, a estimativa é que em 2035 exista cerca de 471 milhões de diabéticos no mundo (SBD, 2016).

A descoberta da insulina em 1921 foi o grande marco da história da DM e a maior conquista para o seu tratamento. Nos últimos 85 anos, desde a descoberta da insulina, houve importantes avanços na insulinoterapia, entre eles, os mais importantes foram a purificação da insulina animal, a substituição pela insulina humana sintética e, mais recentemente, a síntese de análogos de insulina de ação ultrarrápida e prolongada. Todos esses avanços no decorrer do tempo visaram oferecer melhores resultados no controle da patologia (Crisostomo et al., 2017).

No Brasil, mesmo com a existência do Sistema Único de Saúde (SUS), gratuito e universal, o custo individual de uma doença crônica é elevado em função dos custos dos servi- ços e materiais agregados, o que contribui para o empobrecimento das famílias. A necessidade de insulinas análogas de ação rápida e longa já é fator fundamental na vida dos dependentes de insulina, devido a sua ação eficaz durante o processamento do medicamento, tendo considerável melhora na qualidade de vida e socioeconômica do cliente (Mendes, 2012; Brasil, 2016).

A forma de responder socialmente, por meio dos sistemas de atenção à saúde, às condições agudas e crônicas marca as diferenças entre atenção à demanda espontânea e a de atenção programa. Por ser um medicamento de autovalor econômico, os indivíduos que necessitam de análogos de insulina têm duas opções para a obtenção do medicamento: autofinanciar o tratamento medicamentoso ou abrir processo judicial contra os municípios (judicialização) (Mendes, 2012).

A judicialização da saúde brasileira é um fenômeno multifatorial que afeta de maneira prejudicial a execução das políticas de saúde no SUS, uma vez que a determinação judicial para fornecimento de medicamentos acarreta gastos elevados e não previstos nos orçamentos de saúde do estado. Em sua maioria, a judicialização é justificada devido à hipossuficiência financeira do indivíduo que necessite da medicação e do dever e obrigação do estado em fornecer serviços de saúde (Lisboa \& Souza, 2017; Paim et al., 2017).

Apesar dos avanços nas políticas e ações públicas voltadas para a necessidade de atender à demanda dos usuários, é notório que o SUS ainda enfrenta grandes dificuldades para atender à necessidade da população. Ao levar em consideração a Relação Nacional de Medicamentos Essenciais (Rename), tem-se cada vez mais o aumento da demanda judicial por medicamentos não disponibilizados pelo SUS (Santos et al., 2018). Sendo assim, o fenômeno de judicialização para o acesso de medicamentos tem sido objeto de estudo no Brasil (Lisboa \& Souza, 2017).

O baixo poder aquisitivo da população afetada pela DM, a carência de políticas públicas, o fortalecimento do poder judiciário e o amparo constitucional dos direitos humanos são fatores determinantes no amplo processo de crescimento da judicialização da política, ou seja, a forte atuação do judiciário na promoção dos direitos sociais previstos na Constituição Federal de 1988 (Nunes, 2016).

Baseado no aumento dos casos de judicialização para a obtenção de insulinas análogas (e demais medicamentos não fornecidos gratuitamente pelo SUS), associado à ausên- 
cia de trabalhos científicos que contabilizem o custo anual de gasto com esse tipo de solicitação, este estudo afirma sua importância por ser inédito ao demonstrar os gastos anuais com a aquisição de insulina análoga em uma capital do norte do Brasil.

Sendo assim, o presente estudo é relevante e inédito no atual cenário do desenvolvimento da saúde e economia do país, contribuindo para o enriquecimento e a apreciação da comunidade científica. Diante do exposto, este estudo teve como objetivo analisar os gastos com insulinas análogas de ação rápida e longa, no município de Belém/PA, no ano de 2016.

\section{Métodos}

Trata-se de um estudo descritivo, quantitativo de farmacoeconomia, tipo estudo de caso, direcionado aos custos de insulinas análogas utilizadas no tratamento de pacientes com DM, os quais recebem esses fármacos no Centro de Especialidades Médicas e Odontológicas (Cemo), por meio de abertura de processo administrativo iniciado na Secretaria Municipal de Saúde de Belém (Sesma).

Os estudos de farmacoeconomia podem identificar, calcular e comparar custos (recursos consumidos), riscos e benefícios (clínicos, econômicos, humanísticos) de programas ou terapias específicas e determinar quais são as alternativas que produzem os melhores resultados em relação aos recursos investidos (Secoli et al., 2005). Este estudo propõe calcular os gastos que a Sesma teve durante um ano de compras de análogos.

Sabe-se que os custos representam o valor de todos os insumos (trabalho, materiais, pessoal, entre outros) utilizados na produção e distribuição de bens ou serviços. Eles representam o elemento comum dos métodos da farmacoeconomia, que engloba os recursos considerados relevantes na aplicação do tratamento. São classificados em direto, indireto e intangível (Secoli et al., 2005). Nessa perspectiva, adotamos o custo direto.

São quatro os tipos de análises recomendados pela farmacoeconomia: minimização de custos, custo-benefício, custo-efetividade e custo-utilidade. Neste estudo, foi adotada a primeira, pois é a forma mais simples de avaliação econômica, haja vista que somente os custos são submetidos a comparações, pois as eficácias ou efetividades das alternativas variáveis são semelhantes.

Esses usuários têm acesso a esse tipo de medicamento por meio da judicialização, em que existe uma ação civil pública em Belém a favor da Associação dos Diabéticos, representando gastos para a administração pública municipal. Optou-se por esse tipo de estudo e abordagem pela objetividade oferecida para alcançar o objetivo esperado.

Para realizar a pesquisa, foram utilizados como critérios de inclusão: todas as notas de empenho de insulinas análogas, localizadas na Referência Técnica de Medicamentos da Secretária Municipal de Saúde, referente ao ano de 2016. Fo- ram excluídos da pesquisa: I - Notas de empenho referentes a insulinas não análogas; II - Outros tipos de medicamentos utilizados no tratamento da diabetes; e III - Notas de empenho que não correspondiam ao ano de 2016.

O estudo foi realizado na Sesma, do município de Belém/ PA, no setor de Referência Técnica em Políticas de Medicamentos e Assistência Farmacêutica, do Núcleo de Promoção a Saúde (NUPS), mediante coleta de dados como tipo de insulina e quantidade empenhada, contidos em notas de empenho que estavam arquivadas na Secretaria. A coleta foi realizada nos meses de janeiro e fevereiro de 2017.

Os dados foram coletados por meio de fotografias das notas e tabulados em planilhas no Microsoft ${ }^{\circledR}$ Excel 2010 para facilitar a análise e interpretação dos resultados. Posteriormente, foram analisados e houve o desenvolvimento de tabelas e gráficos com informações necessárias à discussão do tema, facilitando a compreensão dos leitores e fornecendo mais teor científico à produção.

Por ser um estudo que não envolve a participação de seres humanos e nem utiliza dados secundários referentes a usuários, sendo os dados analisados de domínio público pertencentes ao SUS, não há a necessidade de submissão e aprovação de Comitê de Ética em Pesquisa (CEP), segundo a Portaria no 466/2012.

\section{Resultados}

Atualmente, a Rename de 2017 inclui apenas a insulina humana NPH (de ação intermediária) e a insulina humana regular (de ação rápida), consequentemente, na Relação Municipal de Medicamentos Essenciais de Belém (Remume), publicada em 07 de março de 2018; há somente a presença dessas duas, não sendo disponibilizadas as análogas de ação ultrarrápida (Aspart, Lispro e Glulisina), de longa ação (Detemir e Glargina) e as pré-misturadas (Regular 30\% + 70\% de NPH; Insulina UItrarrápida 70\% + NPH 30\%). Contudo, elas estão disponíveis no mercado farmacêutico brasileiro, por isso são constantes alvos de judicialização (Brasil, 2014; Lisboa \& Souza, 2017).

Na Tabela 1, são listadas as insulinas análogas fornecidas pela Sesma, no ano de 2016, por meio de judicialização, assim como a quantidade contida em cada frasco. No total, foram disponibilizadas 15 variedades de análogos de insulina, com variação de frascos entre 3 e $10 \mathrm{ml}$.

No ano do presente estudo, foram identificadas 10 notas de empenho referentes à compra de insulina análoga pela Sesma, as quais foram organizadas de acordo com sua data de emissão, número do empenho, tipo de insulina, quantidade, valor unitário e valor total (Tabela 2). A partir desses dados, é possível verificar a variação de valores entre os diversos tipos de apresentação das insulinas, variando de um valor mínimo de $\mathrm{R} \$$ 16,78 (Apidra e Apidra Solostar) até o máximo de R\$ 210,19 (Glargina). 
Durante o ano, foram realizadas 10 compras (10 notas de empenho), com maior número de frascos solicitados na quarta compra do ano (Empenho no 006742/2016), realizada no dia 04/05/2016, totalizando 8.100 frascos adquiridos das insulinas Glargina (6.500) e Apidra (1.600). Em relação ao valor gasto, o mesmo empenho apresentou maior gasto com recursos financeiros, acarretando despesa de $\mathrm{R} \$ 442.232,00$ ao município.
No total, foram adquiridos 30.450 frascos de análogos de insulina (Gráfico 1). A insulina Glargina liderou a quantidade final de compras com 12.650 frascos (41,5\%), seguida da Lispro com 5.000 frascos (16,4\%), Degludeca com 4.000 frascos (13,1\%), Aspart com 3.300 frascos (10,8\%), Apidra com 3.100 frascos (10,2\%), Glulisina com 1.000 frascos (3,3\%), Detemir com 800 frascos (2,6\%) e Liraglutida com 600 frascos (2\%).

Tabela 1. Insulinas análogas disponibilizadas pela Sesma no ano de 2016

\begin{tabular}{|c|c|c|}
\hline Item & Descrição & Quantidade \\
\hline 02 & Insulina Aspart - $100 \mathrm{UI} / \mathrm{ml}$, solução injetável - Sistema aplicador, tipo preenchido e descartável & Frasco $-3 \mathrm{ml}$ \\
\hline 04 & Insulina Detemir - 100 UI/ml, solução injetável - Sistema aplicador, tipo preenchido e descartável & Frasco $-3 \mathrm{ml}$ \\
\hline 05 & Insulina Glargina, dosagem 100 UI/ml, solução injetável & Frasco $-10 \mathrm{ml}$ \\
\hline 07 & Insulina Lispro, dosagem $100 \mathrm{UI} / \mathrm{ml}$, solução injetável & Frasco $-10 \mathrm{ml}$ \\
\hline 08 & Insulina Lispro - 100 UI/ml, solução injetável - Sistema aplicador, tipo preenchido e descartável & Frasco $-3 \mathrm{ml}$ \\
\hline 09 & Insulina Glulisina, dosagem 100 UI/ml, solução injetável & Frasco $-10 \mathrm{ml}$ \\
\hline 10 & Insulina Glulisina - 100 UI/ml, solução injetável - Sistema aplicador, tipo preenchido e descartável & Frasco $-3 \mathrm{ml}$ \\
\hline 13 & $\begin{array}{l}\text { Insulina Lispro 50\% + Lispro Protamina 50\% - } 100 \mathrm{UI} / \mathrm{ml} \text {, solução injetável - Sistema aplicador, } \\
\text { tipo preenchido e descartável }\end{array}$ & Frasco $-3 \mathrm{ml}$ \\
\hline 14 & Insulina Liraglutida - 6 mg/ml, solução injetável - Sistema aplicação multidose, tipo preenchido e descartável & Frasco $-3 \mathrm{ml}$ \\
\hline 15 & Insulina Degludeca - $100 \mathrm{UI} / \mathrm{ml}$, solução injetável - Sistema de aplicação preenchido e descartável & Frasco $-3 \mathrm{ml}$ \\
\hline
\end{tabular}

Fonte: Autores da pesquisa, 2018.
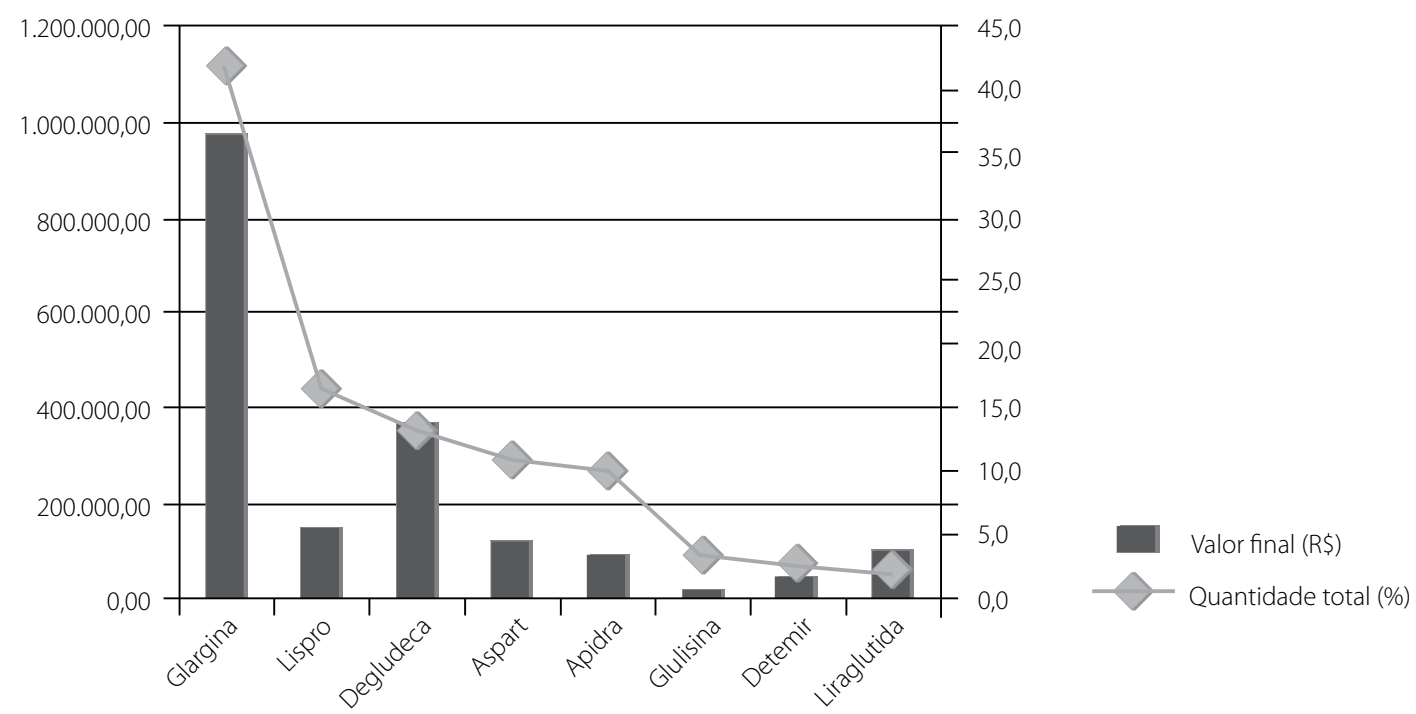

Figura 1. Relação entre quantidade total em porcentagem (\%) e valor gasto em reais (R\$) referente à compra de insulina análoga no ano de 2016. Fonte: Autores da pesquisa, 2018. 
Em relação ao custo com insulinas análogas, o município teve uma despesa total de R\$1.857.778,00 (Gráfico 1). Nesse quesito, a Glargina também liderou o ranking de gastos com R\$ 967.970,00 (52,1\%), Degludeca com R\$363.480,00 (19,6\%), Lispro com R\$ 147.975,00 (8\%), Aspart com R\$120.415,00 (6,5\%), Liraglutida com R\$1 102.000,00 (5,5\%), Apidra com R\$ 91.158,00 (4,9\%), Detemir com R\$ 48.000,00 (2,6\%) e Glulisina com R\$ 16.780,00 (0,9\%).

Sabe-se que o município de Belém apresenta uma particularidade em relação à disponibilização de análogos de insulina. Segundo a Ação Civil Pública n 0006454-87.2008.4.01.3900, de
05/04/2016, o município tem a obrigação de, quando necessário e comprovado mediante laudo médico, fornecer a pacientes diabéticos munícipes de Belém os análogos de insulinas. Para tanto, o usuário deve dar entrada em um processo administrativo na Sesma, o qual será avaliado pela Referência Técnica de Medicamentos e Assistência Farmacêutica, caso seja deferido, ele será encaminhado ao CEMO, onde o paciente receberá os análogos mensalmente (MPF-PA, 2016). Entretanto, os gastos com a aquisição de análogos de insulina por judicialização ainda são altos no município.

Tabela 2. Compra de medicamentos (insulinas análogas)

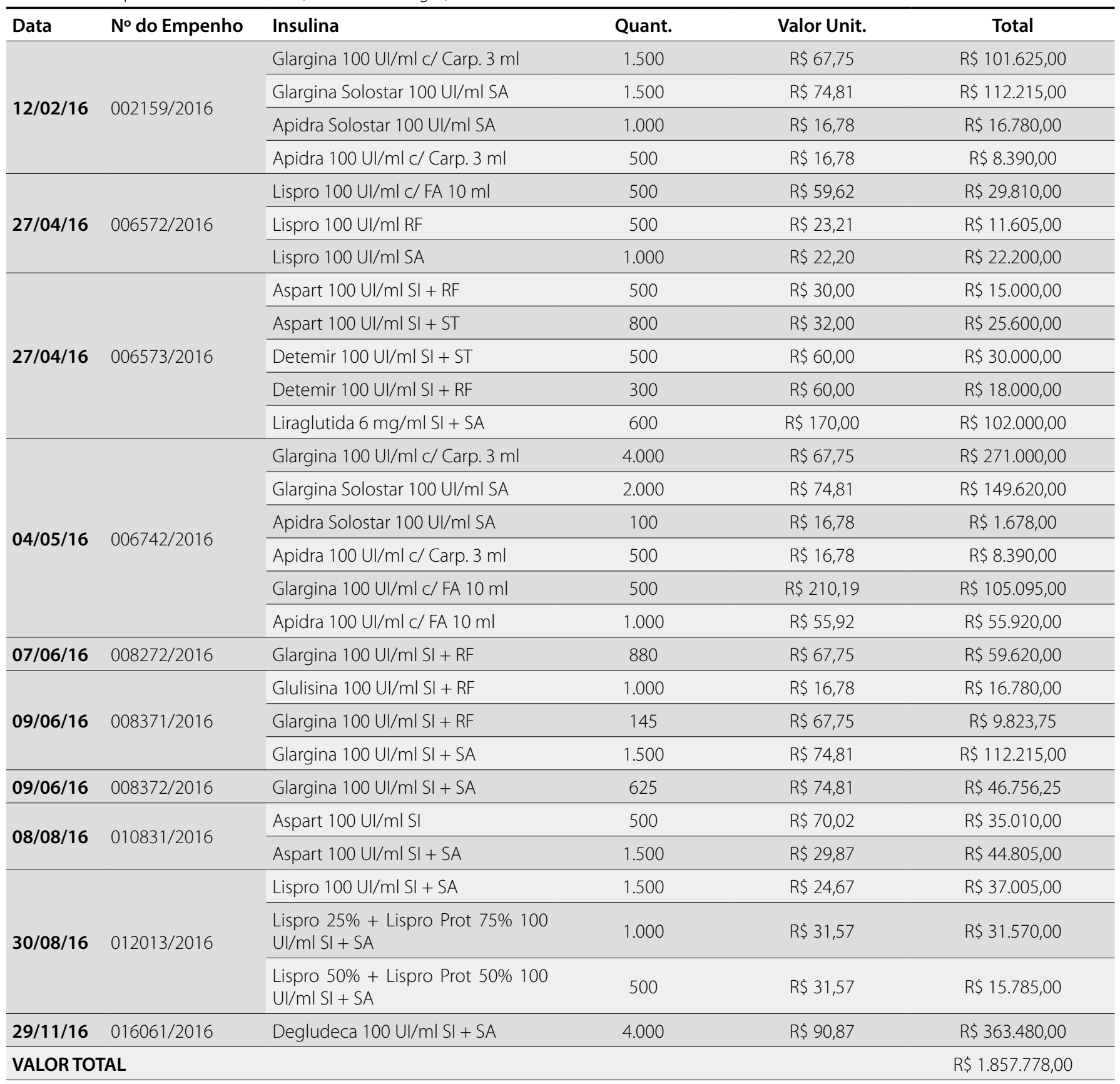

Fonte: Autores da pesquisa, 2018. 


\section{Discussão}

Segundo os dados coletados, a insulina Glargina foi a análoga com maior número de frascos comprados (12.650) e também liderou os gastos com a compra de insulina chegando a quase 1 milhão de reais (R\$976.778,00). Tais dados convergem com estudos realizados no país que demonstram que a insulina Glargina aparece entre os três medicamentos mais solicitados nos estados de São Paulo, Minas Gerais, Santa Catarina (entre 2003 e 2004) e Pernambuco (2009 e 2010) (Chieffi \& Barata, 2010; Campos Neto et al., 2012; Pereira et al., 2010; Marçal, 2012). Em contrapartida, o estudo de Lima (2012) comprovou que no estado do Rio de Janeiro a insulina Aspart foi o medicamento mais solicitado judicialmente nos anos de 2009 e 2010.

A soma dos gastos das insulinas análogas, de duração prolongada - Glargina, Liraglutida, Degludeca e Detemir - atingiu aproximadamente 1,5 milhão de reais ( $\mathrm{R} \$ 1.481 .450,00$ ), concluindo que os gastos com as insulinas análogas de duração prolongada representaram mais de $3 / 4$ das despesas, enquanto as demais insulinas ocuparam menos de $1 / 4$, contribuindo com $R \$ 376.328,00$ nas despesas no ano de 2016.

Em estudo realizado por Lisboa e Souza (2017) no estado da Bahia, foi comprovado o crescimento da demanda de insulinas análogas por via judicial entre os anos de 2010 e 2012, seguindo a tendência de anos anteriores. Contudo, a partir do ano de 2013, houve relativa queda em relação ao ano anterior, explicada pela incorporação das insulinas análogas pela Secretaria de Saúde do Estado da Bahia.

Em consonância com o resultado desse estudo no qual a Glargina é o análogo de insulina com maior número de compra no ano de 2016 (12.650 frascos, 41,5\%). Santos (2018) afirma que $62,4 \%$ dos processos de judicialização são referentes a pedidos de insulinas análogas e que as mais solicitadas são a Glargina e a Detemir. Lisboa (2015) encontra dados semelhantes e muito significativos ao afirmar que Glargina e Aspart representam 91\% das insulinas fornecidas por judicialização no estado da Bahia.

De acordo com a Portaria no 10, de 21 de fevereiro de 2017, foi acatada a recomendação da Comissão Nacional de Incorporação de Tecnologias no SUS (Conitec), referente à incorporação dos análogos de ação rápida ao SUS para o tratamento de pessoas com DM tipo 1. Com base na Lei no 12.401/2011 e no Decreto no 7.646/2011, que regula o funcionamento da Conitec e processo de incorporação de tecnologias ao SUS, o Ministério da Saúde tem 180 dias (a partir da publicação da decisão no Diário Oficial da União) para disponibilizar a tecnologia no SUS.

Segundo o Decreto no 7.508/2011, todo e qualquer fármaco incorporado às tecnologias do SUS devem ser incluídos na Rename para sua prescrição, dispensação e utilização na rede pública de saúde. Entretanto, as insulinas ainda não constam na Rename, portanto não foram incluídas na Relação Munici- pal de Medicamentos Essenciais (Remume) do munícipio de Belém/PA.

Mesmo com todos os avanços tecnológicos, ainda hoje os gastos com tratamento de portadores de DM são altos, exigindo boas condições financeiras dos portadores da doença para tratamento adequado da patologia. Segundo o Instituto de Pesquisa Econômica Aplicada (Ipea), as despesas com medicações são o principal componente do gasto com saúde entre as famílias brasileiras (Ipea, 2013; Lisboa \& Souza, 2017). Devido ao alto custo das insulinas análogas, Nunes (2016) conclui que os medicamentos e insumos para o tratamento de clientes portadores de DM são uma das mais importantes causas dos processos de judicialização.

Devido à ausência de protocolo que padronize o processo de acesso a esses fármacos, percebe-se o pouco rigor na avaliação dos processos de pedido de análogos, banalizando o acesso e, assim, aumentando consideravelmente os gastos do município com as insulinas, apresentando-se como um dos principais custos relacionados a medicamentos na Sesma.

\section{Conclusão}

A judicialização da saúde no Brasil, entre todas as suas consequências, conseguiu trazer o tema do efetivo cumprimento do direito fundamental à saúde para o centro do debate nas instituições gestoras das políticas públicas de saúde. Entretanto, a judicialização contribui para grandes desafios para as esferas municipais, estaduais e federais, uma vez que gera uma demanda de gastos imprevisíveis.

Quanto aos gastos com aquisição de análogos de insulinas realizados pelo município de Belém no ano de 2016, fica claro que há maior demanda e maiores gastos com a insulina Glargina. Mesmo com a incorporação de algumas insulinas análogas na Rename, a Glargina não foi contemplada na lista de inclusão, por isso estima-se que as despesas extras para a obtenção desses medicamentos no município ainda sejam significativas nos anos seguintes.

A partir da identificação das insulinas análogas mais solicitadas por meio de judicialização e da obtenção do custo anual na compra desses medicamentos, é possível fazer estimativas de gastos financeiros advindos dos fundos municipais de saúde e, assim, investir em planejamento em saúde. Logo, a realização de estudos semelhantes a este podem fornecer subsídios para os municípios realizarem, por exemplo, a inclusão desses análogos na Remume relativamente à demanda que eles recebem, favorecendo a prestação de serviços de saúde que respeitem os princípios do SUS e da Constituição Federal.

Os gastos com a aquisição de medicamentos por meio da judicialização constituem uma das grandes fontes de despesas extras que o município tem durante o ano. Futuramente, com a obrigatoriedade da disponibilização de alguns tipos de insulinas análogas (que devem constar na Rename) por meio 
dos serviços de saúde do SUS, espera-se que haja diminuição gradativa da judicialização e, consequentemente, redução dos gastos do munícipio na aquisição de insulinas análogas.

Devido à falta de estudos dessa natureza, faz-se necessária a realização de estudos e discussões que abordem a temática da farmacoeconomia e vantagens e desvantagens relacionadas à aquisição de análogos de insulina pelo SUS, a fim de fornecer informações essenciais para assistência, planejamento e gestão dos serviços de saúde. Por tratar-se de um estudo inédito, este servirá como subsídio, referência e norteamento para futuros estudos nessa linha de pesquisa.

\section{Agradecimentos}

Agradecemos à Secretaria de Saúde do Município de Belém (Sesma) pela disponibilização das notas de empenho para a realização da pesquisa.

\section{Referências bibliográficas}

Brasil. Ministério da Saúde. Comissão Nacional de Incorporação de Tecnologias no SUS. Secretária de Ciência, Tecnologia e Insumos Estratégicos. Insulinas análogas de ação rápida para Diabetes Mellitus Tipo 1. Brasília-DF; 2016

Brasil. Ministério da Saúde. Departamento de Gestão e Incorporação de Tecnologias em Saúde da Secretaria de Ciência, Tecnologia e Insumos Estratégicos - DGITS/SCTIE. Comissão Nacional de Incorporação de Tecnologias no SUS (Conitec) - Relatório n 103 - Insulinas análogas de longa ação para diabetes mellitus tipo II. Brasília-DF; 2014.

Brasil. Presidência da República. Decreto no 7.508, de 28 de junho de 2011. Regulamenta a Lei no 8.080, de 19 de setembro de 1990, para dispor sobre a organização do Sistema Único de Saúde - SUS, o planejamento da saúde, a assistência à saúde e a articulação interfederativa, e dá outras providências. Brasília-DF, 2011. Available in: http://www.planalto.gov.br/ ccivil_03/_ato2011-2014/2011/decreto/D7508.htm. Accessed on: 12/12/2018.

Brasil. Presidência da República. Decreto no 7.646, de 21 de dezembro de 2011. Dispõe sobre a Comissão Nacional de Incorporação de Tecnologias no Sistema Único de Saúde e sobre o processo administrativo para incorporação, exclusão e alteração de tecnologias em saúde pelo Sistema Único de Saúde - SUS, e dá outras providências. Brasília-DF; 2011. Available in: http://portal.saude.gov.br/portal/arquivos/pdf/ DECRETO_7646_CONITEC.pdf. Accessed on: 12/12/2018.

Brasil. Presidência da República. Lei no 12.401, de 28 de abril de 2011. Altera a Lei no 8.080, de 19 de setembro de 1990, para dispor sobre a assistência terapêutica e a incorporação de tecnologia em saúde no âmbito do Sistema Único de Saúde - SUS. Brasília-DF; 2011. Available in: http://www. planalto.gov.br/ccivil_03/_Ato2011-2014/2011/Lei/L12401.htm. Accessed on: 12/12/2018.

Brasil. Presidência da República. Portaria no 10, de 21 de fevereiro de 2017. Torna publica a decisão de incorporar insulina análoga de ação rápida para o tratamento de Diabetes Mellitus Tipo 1, no âmbito do Sistema Único de Saúde - SUS. Brasília-DF, 2017. Available in: http://conitec.gov.br/ images/Relatorios/Portaria/2017/PortariasSCTIE-09e10_2017.pdf. Accessed on: 12/12/2018.
Campos Neto OH, Acurcio FA, Machado MAA, Ferré F, Barbosa FLV, Cherchiglia ML, et al. Médicos, advogados e indústria farmacêutica na judicialização da saúde em Minas Gerais, Brasil. Rev Saúde Pública. 2012;46(5):784-90.

Chieffi AL, Barata RCB. Ações judiciais: estratégia da indústria farmacêutica para introdução de novos medicamentos. Rev Saúde Pública. 2010;44(3):421-9.

Crisóstomo IS, Souza JS, Mantovani JT, Castro GFP. A insulinoterapia e a Atenção Farmacêutica aos portadores de diabetes mellitus tipo I. Revista Transformar. Itaperuna-RJ/10a edição/2017/1.

Henriques RS. Custos do tratamento do paciente diabético tipo 2 sob a perspectiva do SUS [dissertação]. Curitiba: Universidade Federal do Paraná; 2016.

IDF - International Diabetes Federation. Diabetes Atlas. 7th ed. Brussels: IDF; 2015.

Ipea - Instituto de Pesquisa Econômica Aplicada. Dimensões do acesso a medicamentos no Brasil: perfil e desigualdades dos gastos das famílias, segundo as pesquisas de orçamentos familiares 2002-2003 e 2008-2009. Brasília: Ipea; 2013.

Lima GS. Demanda judicial de medicamentos e uso de indicadores de avaliação e monitoramento no estado do Rio de janeiro [dissertação]. Rio de Janeiro: Fundação Oswaldo Cruz; 2012

Lisboa ES. Acesso ao tratamento da diabetes na Bahia: por que se recorre ao judiciário? [dissertação]. Salvador: Universidade Federal da Bahia; 2015.

Marçal KKS. A judicialização da assistência farmacêutica: o caso de Pernambuco em 2009 e 2010 [dissertação]. Recife: Fundação Oswaldo Cruz; 2012.

Mendes EV. O cuidado das condições crônicas na atenção primária à saúde: O imperativo da consolidação da Estratégia da Saúde da Família. Brasília: Opas; 2012

Ministério Público Federal do Estado do Pará - MPF/PA. Poder Judiciário. Tribunal Regional Federal da Primeira Região. Seção Judiciária do Estado do Pará. Processo nº 0006454-87.2009.4.01.3900 - 1a Vara Federal. BelémPA, 2016. Available in: http://biblioteca.mpsp.mp.br/PHL_IMG_PORTAL/ Bjuris/bjuris_abr-2016.html. Accessed on: 12/12/2018.

Nunes RP. Judicialização no âmbito do Sistema Único de Saúde: um estudo descritivo sobre o custo das ações judiciais na saúde pública do município de Juiz de Fora [dissertação]. Juiz de Fora: Faculdade de Medicina da Universidade Federal de Juiz de Fora; 2016.

Pereira JR, Santos RI, Nascimento Júnior JM, Schenkel EP. Análise das demandas judiciais para o fornecimento de medicamentos pela Secretaria de Estado da Saúde de Santa Catarina nos anos de 2003 e 2004. Ciênc Saúde Coletiva. 2010;15(Supl 3):3551-60.

Santos ECB, Teixeira CRS, Zanetti ML, Istilli PT, Pereira LHTR, Torquato MTCG. Judicialização da saúde: acesso ao tratamento de usuários com diabetes mellitus. Texto Contexto Enferm. 2018;27(1):e0800016.

Secoli SR, Padilha KG, Litivoc J, Maeda ST. Farmacoeconomia: perspectiva emergente no processo de tomada de decisão. Ciênc Saúde Coletiva. 2005;10(Supl):287-96.

Smeltzer SC, Bare BG. Brunner \& Suddarth: Tratado de Enfermagem MédicoCirúrgica. 12a ed. Rio de Janeiro: Guanabara Koogan; 2014. v. I e ll.

SBD - Sociedade Brasileira de Diabetes. Diretrizes da Sociedade Brasileira de Diabetes 2015-2016. São Paulo: A.C. Farmacêutica; 2016. 